\title{
Excision of a chondroma from the plantar foot accompanied by application of an external continuous tissue expander to facilitate wound closure: a case report
}

\begin{abstract}
Extra skeletal chondromas, also known as chondromas of the soft parts, are benign cartilaginous tumors which can arise de novo from soft tissue. They are extremely rare with a predilection for the extremities, particularly the hands and the feet. A review of the published data reveals ample literature highlighting the recognition and management of these kinds of tumors involving the sole of the foot. However, there is limited data addressing the facility of wound closure in the presence of a large soft tissue defect that can potentially be the result of tumor excision. We discuss our findings in the context of a case of a 70 year old male, who presented with such an anomaly involving the plantar aspect of his right foot. Biopsy of the lesion prior to surgery was consistent with a chondroma. We present a dual approach to treatment of this condition involving both the excision and simultaneous wound revision process by utilizing a Derma $\operatorname{Close}^{\circledR}$ tissue expander. With the aid of this device, we were able to excise the soft tissue mass and primarily close our surgical site by minimizing the tension at the wound edges.
\end{abstract}

Keywords: Tissue approximation device, Extraskeletal chondroma, Chondroma of soft parts, primary tumors of the foot, DermaClose ${ }^{\circledR}$
Volume 6 Issue 3 - 2016

\author{
Michael Subik,' Rahul Bhatt ${ }^{2}$ \\ 'Private Practice in Dallas, USA \\ ${ }^{2}$ Director of North Jersey Reconstructive Foot and Ankle \\ Fellowship, USA
}

Correspondence: Rahul Bhatt, DPM,AACFAS, 3324 McKinney Ave, Dallas, TX 75204, USA, Tel 3I5-794-4978, Fax 8I7-460I307, Email rbhattny@gmail.com

Received: November 03, 2016 | Published: November 03, 2016
Abbreviations: ESC, Extra Skeletal Chondroma; ESMCS, Extraskeletal Myxoid Chondrosarcomas

\section{Introduction}

Extra skeletal chondroma (ESC), also referred to as chondroma of soft parts, is a benign soft tissue tumor composed mainly of hyaline cartilage with no connection to bone or periosteum. It is a very rare, slow progressing, benign tumor with a predilection for hands and feet. It can sometimes be misdiagnosed as a synovial cyst or a more serious condition such as synovial sarcoma. These tumors are usually small, measuring less than $3 \mathrm{~cm}$ in diameter and are histologically similar to juxtacortical or periosteal chondromas, which are located between the bone and periosteum. The most frequent sites of involvement for an ESC are the distal extremities. Less commonly, it is found in the oral cavity, pharynx, trunk, and knee. ESC is a lesion that can be found at any age, but it usually occurs in adults and is very rare in children with only a few case reports in the literature. ${ }^{2}$ Moreover, it can exhibit worrisome radiologic and histological features mimicking chondrosarcomas. In the case of our patient, pathological analysis by fine needle aspiration biopsy confirmed that the lesion was indeed a chondroma and thereby a benign lesion. Subsequently, the decision was made to proceed with resection of the tumor with simultaneous application of the DermaClose ${ }^{\circledR}$ (Wound Care Technologies Incorporated, Chanhassen, Minnesota, United States) continuous external tissue expander to facilitate wound closure. We decided to use such a device given the size of the lesion and the anticipated dead space that would ensue following excision of the tumor. Significant edema of the skin and surrounding soft tissues was also present, which could have been hindrance to wound closure. Furthermore, the patient's advanced age, history of diabetes mellitus, and desire to be ambulatory as soon as possible created concern that he may not be entirely compliant with postoperative non-weight bearing instructions.
In the present report, we describe a single stage process that addresses not only the excision of a rare pedal lesion, but the concomitant soft tissue defect management process as well.

\section{Case presentation}

This is the case of a 70 year old male who was treated by us from 9/2015 to $2 / 2016$. The patient, who had no known allergies, had a past medical history of Insulin Dependent Diabetes Mellitus, prostate cancer, and renal cell carcinoma, was referred to our service by an orthopedic colleague. The patient complained that he began noticing a swelling 6 months prior on the plantar medial arch of his right foot. As per the patient, the mass continued to increase in size leading to pain for which the biopsy was taken on 9/9/2015, confirming the diagnosis of chondroma. Since then, the patient had pain upon initial standing and some discomfort while walking. Approximately 2-3 days prior to admission, the patient noticed swelling and redness to the plantar surface. On the day of admission, patient presented with right foot cellulitis, chills, and a temperature of $103 \mathrm{~F}$. Upon examination, a firm, non-mobile swelling was present in the plantar arch of the right foot with a central area of ulceration measuring roughly $3 \mathrm{~cm}$ in diameter. On palpation, the swollen area was found to be excessively warm and tender. The ulcer was superficial with well-defined margins and no veins were dilated. No distal neurovascular deficit or defined proximal lymphadenopathy was present. The blood investigations revealed a hemoglobin of $13.8 \mathrm{~g} / \mathrm{dl}$, leukocytosis with a white blood cell count of 14,900 , and erythrocyte sedimentation rate of $3 \mathrm{~mm}$ at 1 hour. Culture of the wound was positive for methicillin sensitive Staphylococcus aureus. Plain radiographs of the foot, both lateral and oblique views, revealed a radiodense extraskeletal mass in the plantar arch that did not seem to distort the normal osseous anatomy of the right foot (Figure 1). Computed Tomography imaging was performed to evaluate the size and extent of the lesion, which was found to be contiguous with the 
plantar fascia. The approximate size was estimated to be $2.9 \times 6.2 \times 4.0$ $\mathrm{cm}\left(71.92 \mathrm{~cm}^{3}\right)$. Surgical removal of the mass was planned after the resolution of infection. Infectious Disease was consulted and the patient was treated with intravenous Ceftriaxone $1 \mathrm{gm}$ daily (following the initial single doses of intravenous Vancomycin $1 \mathrm{gm}$ and Zosyn $4.5 \mathrm{gm}$ upon admission) and was evaluated daily for the control of infection. Once the patient was optimized for surgery, we began our procedure as follows: the patient was positioned in the supine position under general anesthesia. The foot was exsanguinated and an ankle tourniquet was inflated to $250 \mathrm{~mm} / \mathrm{Hg}$. The plantar wound was found to measure roughly $3 \mathrm{~cm}$ in diameter. Incisions were made taking $0.5 \mathrm{~cm}$ margins on both the medial and lateral sides equally. A fusiform excision technique was used which resulted in the creation of a wound that was $4 \mathrm{~cm}$ wide and $12 \mathrm{~cm}$ long. Using meticulous blunt and sharp dissection, the redundant wedge of skin was excised, including the wound itself. A large chondral mass was subsequently encountered, which was contiguous with the deep and plantar fascia and approximately $9 \times 4 \times 3 \mathrm{~cm}\left(108 \mathrm{~cm}^{3}\right)$ in dimension (Figure 2). As it was in close proximity to the deep neurovascular structures, a small sensory branch of lateral plantar nerve was sacrificed. After thorough excision of the mass, the surgical site was copiously irrigated with 50,000 units of a Bacitracin and Polymyxin solution. The deep plantar fascia was repaired using 2-0 Vicryl suture. The wound was then approximated using the DermaClose ${ }^{\circledR}$ tissue expander to decrease the tension on the surrounding skin and subcutaneous tissue. Given the large size of the defect, soft tissue edema, and patient's history of diabetes, it was deemed necessary to utilize the device. Application began with the placement of three anchors on the medial and distal sides of the incision. The anchors were applied roughly $1 \mathrm{~cm}$ from the wound margins and $1.5 \mathrm{~cm}$ from each adjacent anchor, and were subsequently secured with skin staples. The appropriate, associated lacing technique of the corresponding tension line was then employed. Tension was applied by turning the knob on the device to the desired position, thus pulling the line through a tube system, which was placed across the medial arch.
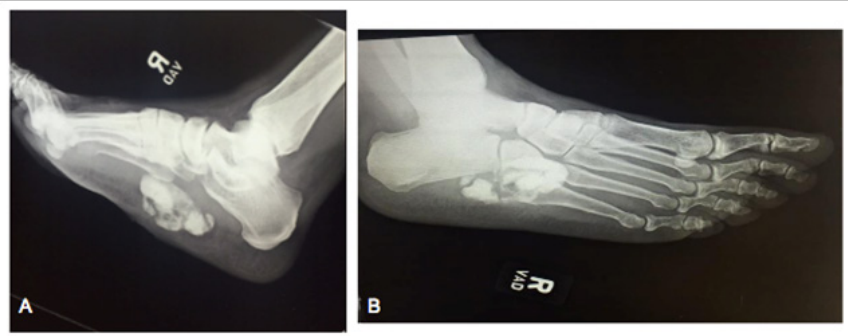

Figure I Preoperative medial oblique (A) and lateral (B) radiographic views demonstrating a radiopaque mass of the plantar midfoot with no bony involvement.

This allowed the device to be affixed with a single 2.0 silk suture to the dorsal medial aspect of the foot and away from the weight bearing surface. The central portion of the wound was left slightly open to drain. Interposed 2.0 Prolene trauma sutures were then placed to further strengthen the re-approximation. Following completion of the procedure, the tourniquet was deflated and hemostasis was maintained.

A bulky dressing with a posterior splint was applied to the surgical site and strict non-weight bearing instructions were given. The patient followed up in our wound care clinic after 1 week, at which time the wound was diminished in size to $8.4 \mathrm{~cm} \times 0.1 \mathrm{~cm}$. The patient continued to follow up once weekly as the wound continued to diminish in size. The device was de-tensioned and removed under posterior tibial nerve block after 3 weeks. At this time, the patient was also allowed to begin protected weight bearing in a fracture boot. At approximately 8 weeks post -op, the wound had healed satisfactorily and the patient was cleared to ambulate in a sneaker. The patient continued to follow up with us over the next 5 months without any residual symptomatology (Figures 3-8).

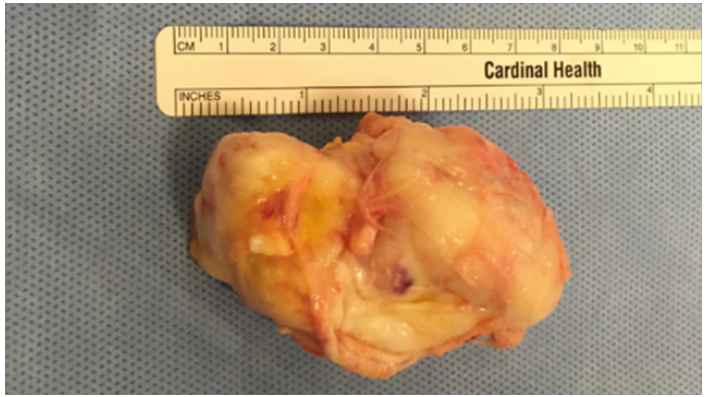

Figure 2 Bilobed, cartilaginous specimen immediately after surgical excision.

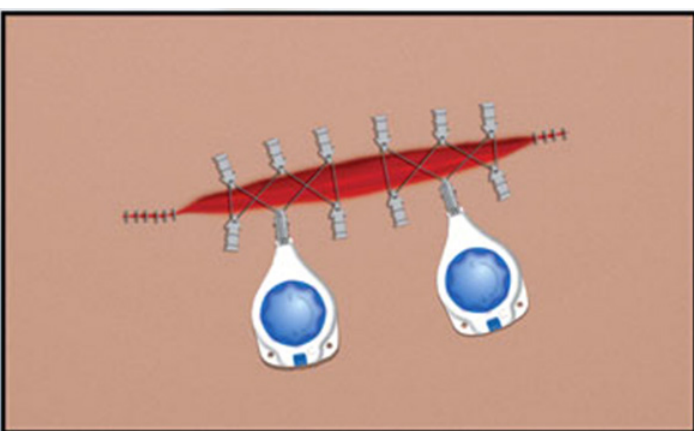

Figure 3 The DermaClose ${ }^{\circledR}$ external tissue expander.

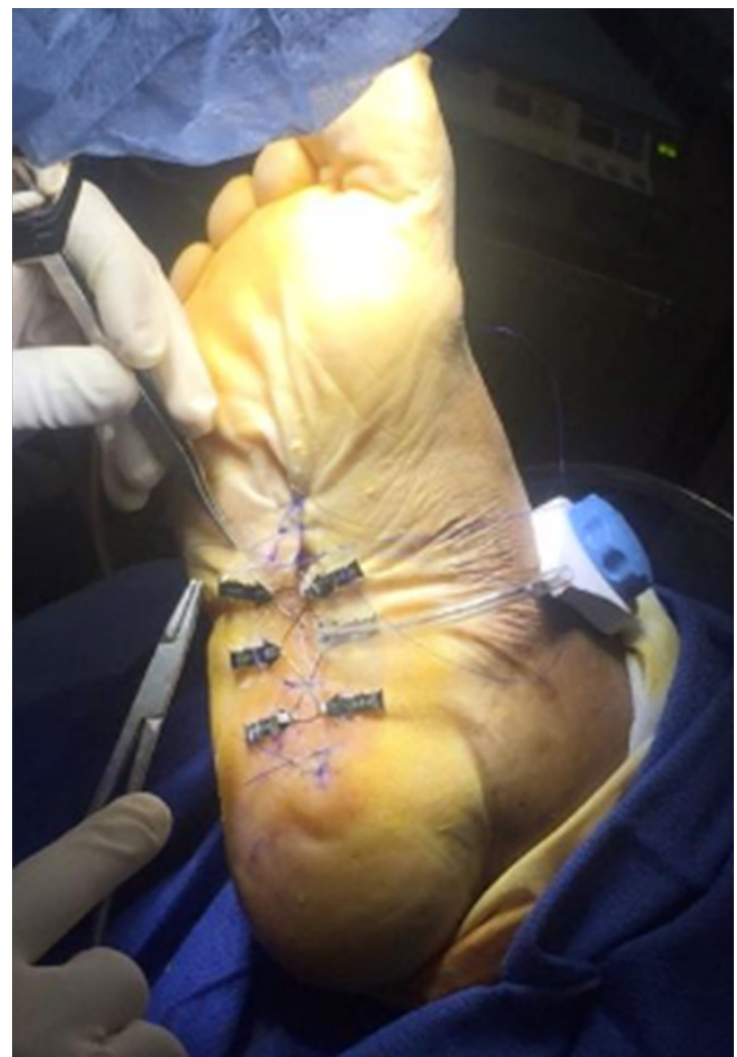

Figure 4 Application of the The DermaClose ${ }^{\circledR}$ immediately after resection of the mass. 

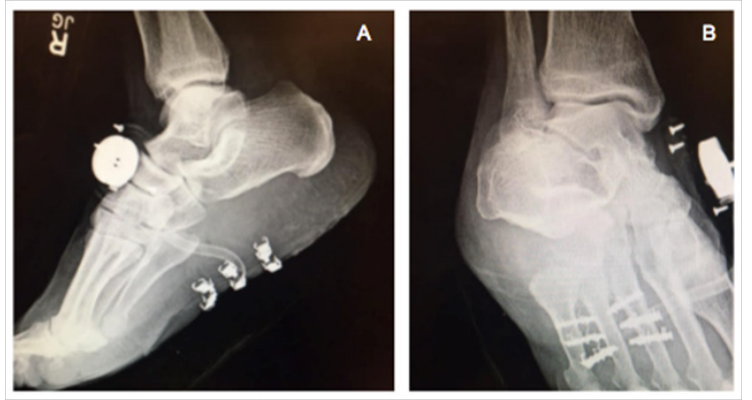

Figure 5 Postoperative medial oblique $(A)$ and lateral $(B)$ radiographic views demonstrating absence of the mass with DermaClose $®$ apparatus in place.

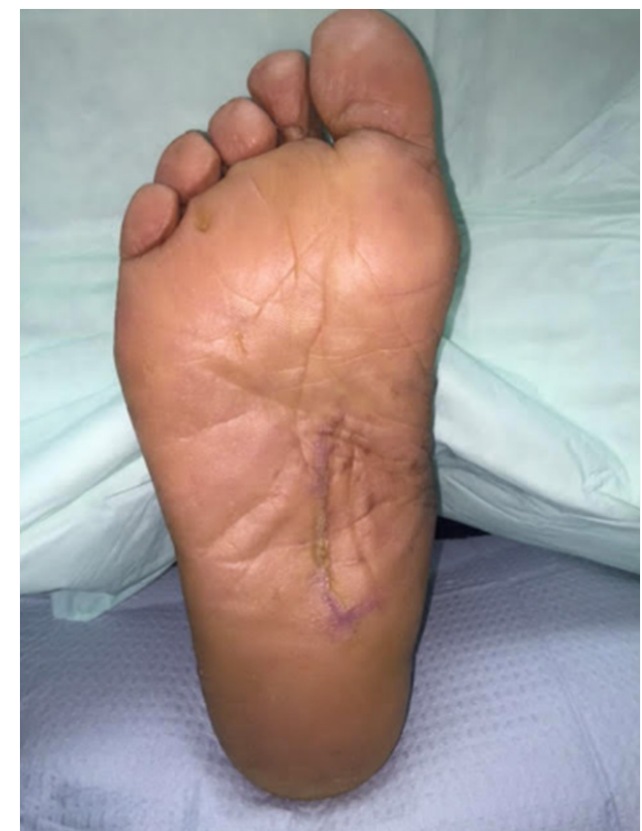

Figure 6 Examination at 5 months after surgery, demonstrating that the wound has healed without any evidence of scar sensitivity or other symptomatology.

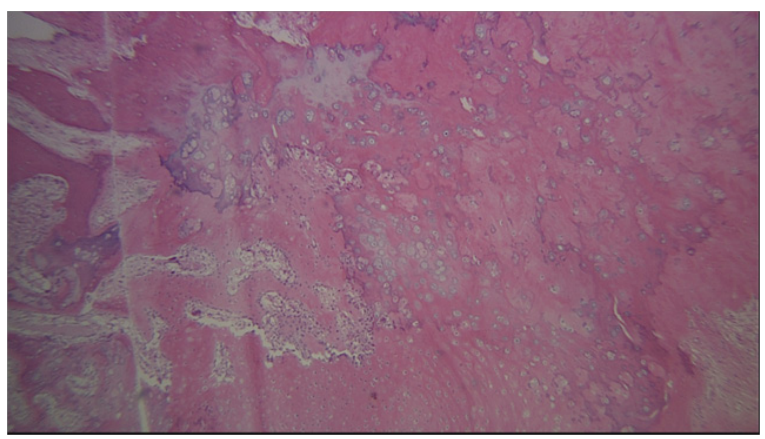

Figure 7 Histomicrograph showing benign chondrocytes adjacent to and admixed with bony spicules. The islands of benign chondrocytes in an area that usually does not have cartilage consitute a chondroma (original magnification 40x, hematoxylin and eosin).

\section{Discussion}

ESC or Soft tissue chondroma is a rare benign cartilaginous tumor and nearly $80 \%$ of ESC's are found in the fingers with the remainder being seen in hands, feet, toes, and trunk. Few cases are reported at rare sites like the dura, larynx, pharynx, oral cavity, skin, fallopian tube, and the parotid gland. It usually arises as a slowly growing, painless mass in adults, with an age range of 30-60 years.

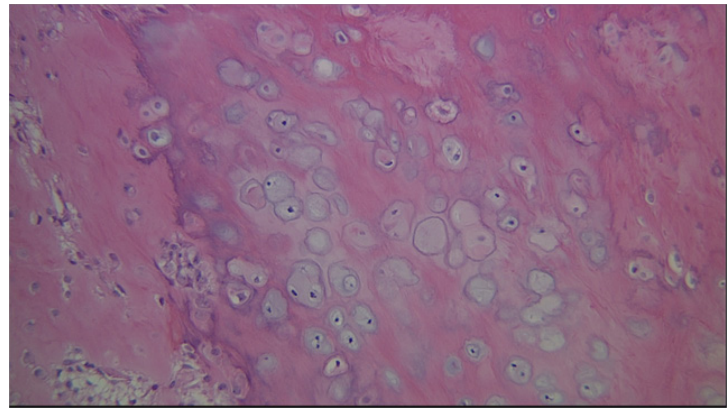

Figure 8 Histomicrograph which shows the chondrocytes more clearly (high power magnification 100x, hematoxylin and eosin).

There is a slight male predominance, as in the case of our patient. It is solitary in nature and may occasionally involve multiple sites, often being associated with tendons, tendon sheath, and joint capsules. Dahlin and Salvadore suggested a synovial origin. ${ }^{3}$ Though it does not involve bones, it may leave compression deformities or bone erosions. Radiographically discrete, irregular, ring like, or curvilinear calcifications are often seen. The lesion appears as well demarcated, firm, rounded small masses, which seldom exceed $3 \mathrm{cms}$ in greatest diameter. ${ }^{4}$ Three variants are typically seen: (a) as multiple nodules of synovial chondromatosis within a joint, (b) as a solitary lesion in association with articulations, within or adjacent to them, and (c) as an isolated cartilaginous lesion in the soft tissues, mostly in the hands and feet. The latter of the two groups of extraskeletal chondromas are rare and frequently exhibit areas of immature cartilage with worrisome histologic features, which could mislead the pathologist to an over diagnosis of chondrosarcoma. ${ }^{5}$

It should be differentiated from benign tumors like ganglion cyst, myositis ossificans, pseudomalignant osseous tumor, ossifying fibromyxoid tumor, synovial chondromatosis, osteochondroma, or a malignant tumor like chondrosarcoma, extraskeletal osteosarcoma and synovial sarcoma. Diagnostic errors can be avoided if any soft tissue lesion that cannot be diagnosed is regarded as potentially malignant until proven otherwise. ${ }^{1}$

Most importantly, ESC's have to be differentiated from Extraskeletal Myxoid Chondrosarcomas (ESMCS). ESC's can be differentiated from ESMCS's, as in our case, by their smaller sizes, characteristic locations in extremities, low cellularities, and the fact that the chondrocytes in the former are better differentiated in the peripheral portions of the tumor. Foci of calcification and ossification are features, which are commonly seen only in ESC's, in contrast to ESMCS's. ${ }^{4}$ ESC's can recur but they never metastasize, whereas ESMCS's can metastasize, which require aggressive treatment. ${ }^{6}$

On conventional radiography, an ESC may appear as a wellcircumscribed, lobulated mass with dense central mineralization. Calcification is usually ring-like, punctate, or granular, suggesting the presence of hyaline cartilage. Sometimes, mineralization has an unusual configuration or is completely absent, as in our case. Computed tomography can confirm the extra skeletal location of the mass and show foci of calcification or ossification that can direct the diagnosis towards ESC. Magnetic Resonance Imaging may delineate the lesion location and margins, but the appearance is nonspecific. In the case of our patient, this was not performed as the patient previously underwent placement of a penile implant. ESC's have been described as showing low to intermediate signal intensity on T1-weighted images and heterogeneous intermediate-to-hyperintense signal from the cartilages on T2-weighted images. ${ }^{2}$ 
DermaClose ${ }^{\circledR}$ is a continuous external device that facilitates rapid tissue movement to reduce or re-approximate wounds. Once the initial application has been completed the device does not require any additional tightening. It is indicated for use in reducing or assisting with the closure of full-thickness wounds of the skin. This tissue expander has enabled a rapid aesthetic delayed primary closure in $93 \%$ of fasciotomy wounds. Its use at Albany Medical Center in Albany, New York has demonstrated cost savings (per wound) of $\$ 4,622$ versus NPWT (VAC) treatment and \$9,117 versus the use of vessel loops. Some of the benefits are as follows. There is reduced time to closure with the average time to closure being 4.4 days (Walter Reed Study in Annals of Plastic Surgery October, 2012). Wounds can be sutured closed in less than 7 days for most procedures. It's a simpler, faster, cheaper alternative to skin grafts and flaps often offering better cosmetic results. It has a broad application for multiple specialties including Plastics, Orthopedics, General Surgery, Vascular Surgery, and Podiatry. ${ }^{7}$

There is limited data to date that employs simultaneous removal of a mass lesion in the foot and application of a tissue expander to address the resulting soft tissue defect. In the management of large complex wounds, external tissue expansion has proven to be a valuable adjunct in achieving definitive wound closure. It can often aid in successful delayed primary closure of certain soft tissue wounds, has low associated morbidities, and can reduce the need for more complex or morbid procedures when used properly. ${ }^{8}$ We believe that this technique can be particularly useful in a myriad of patients, particularly in the elderly, diabetics, and non-compliant patients where there is always concern regarding timely wound healing and possibility of superimposed infection.

\section{Acknowledgements}

We would like to thank Dr. David Gay, third year Pathology resident at Hackensack University Medical Center (Hackensack, New Jersey, USA) for his assistance in providing the histomicrographs that were used in this case report.

\section{Conflicts of interest}

Michael Subik, DPM, FACFAS - Consultant: Stryker Orthopedics.

\section{References}

1. Bahnassy M, Abdul-Khalik H. 2 Soft Tissue Chondroma: A Case Report and Literature Review. Oman Med J. 2009;24(4):296-299.

2. Adaletli I, Laor T, Yin H, et al. Extraskeletal Chondroma: Another Diagnostic Possibility for a Soft Tissue Axillary Mass in an Adolescent. Case Rep Orthop. 2011:309328.

3. Dahlin DC, Salvador AH. Cartilaginous tumors of the soft tissues of the hands and feet. Mayo Clin Proc. 1974;49(10):721-726.

4. Rapini RP, Bolognia JL, Jorizzo JL. Dermatology: 2-Volume Set 2nd. St Louis: Mosby, USA. 2007pp. 2584.

5. Marcial-Seoane RA, Marcial-Seoane MA, Ramos E, et al. Extraskeletal chondromas. Bol Asoc Med P R. 1990;82(9):394-402.

6. Rajalakshmi V, Jayaraman, Anand V, et al. Extraskeletal Chondroma of the Foot-A Case Report. J Clin Diagn Res. 2014;8(3):134-135.

7. DermaClose A Great Alternative To A Skin Graft. 2016

8. Santiago GF, Bograd B, Basile PL, et al. Soft tissue injury management with a continuous external tissue expander. Ann Plast Surg. 2012;69:418-421. 